\title{
The next exoplanet mission to fly
}

\author{
As the CHaracterising ExOPlanet Satellite (CHEOPS) is scheduled for launch later this year, European Space \\ Agency (ESA) Project Scientist Kate Isaak and Principal Investigator Willy Benz give an overview of ESA's first \\ science mission dedicated to the follow-up of known exoplanets orbiting bright stars.
}

T he first planet orbiting a Sun-like star other than our own was discovered more than 20 years ago ${ }^{1}$. Since then, a plethora of exoplanets have been identified that demonstrate that planets and planetary systems are much more diverse than ever thought before. With over 4,000 exoplanets known today, the scientific interest in the field is shifting, transitioning from an era of discovery to one of characterization. With the largest facilities in space (James Webb Space Telescope) or on the ground (extremely large telescopes) on the horizon, detailed studies of a nearby sample of the most interesting planets will soon become possible.

While NASA's Transiting Exoplanet Survey Satellite (TESS) is scanning the whole sky discovering planets that could be suitable for these studies, ESA's CHEOPS (Fig. 1) aims at follow-up studies of the potentially most interesting targets (discovered by TESS or any other search), thereby providing important new constraints on their structure, formation and evolution ${ }^{2}$, while at the same time ensuring an optimum investment of observation time of future large facilities.

CHEOPS will use the technique of ultra-high-precision, broadband transit photometry, monitoring changes in the light from a star as a planet crosses, or transits, its disk. The transit depth is a measure of the square of the ratio of the radii of the planet and the star: hence, combined with a knowledge of the star size yields that of the planet. Targeting already-known planets orbiting bright stars $\left(6 \leq m_{\mathrm{V}} \leq 12\right)$ for which transit times are known means that CHEOPS knows where and when to point, thereby ensuring high efficiency and high accuracy. If needed (for instance, for smallsized planets), the measurement precision can be increased by repeating the transit observation several times.

The very high photometric precision and stability required of CHEOPS are challenging to achieve - the depth of the transit of an Earth-sized planet transiting a Sun-sized star is around 100 parts per million (p.p.m.), setting a floor of a couple of tens of p.p.m. if really constraining information is to be obtained from these measurements. This stringent requirement - to be achieved on timescales of a few hours, and to be maintained for much longer - has driven many aspects of the mission design.

The payload of CHEOPS consists of a single instrument: an ultra-high-precision photometer operating over 330-1,100 nm (ref. $\left.{ }^{3}\right)$. Starlight is collected by a 30-cm-diameter, clear aperture RitcheyChrétien telescope $(f / 5)$ with a single, back-illuminated, low-noise $1,024 \times 1,024$ pixel charge-coupled device (e2v CCD42-70 AIMO) in its focal plane. A baffle system shields the detector from sources of stray light. The telescope is deliberately defocused to minimize the impacts of variations in the responses of individual CCD pixels and jitter in the spacecraft pointing.

CHEOPS is a small-class mission in ESA's science programme, developed and built in partnership with Switzerland (Swiss Space Office). The 'small' in small-class translates to a cost of no more than $€ 50$ million to ESA, with a short development and implementation phase (4-5 year duration), and a very different distribution of responsibilities from typical ESA science missions ${ }^{4}$. The small-mission class provides additional ESA member states - here, Switzerland - with the opportunity to lead the mission consortium, and work with ESA to realize the mission. ESA is the mission architect and procures the launch opportunity and the spacecraft, in this case from Airbus, while the payload is provided by the mission consortium. Unlike for larger missions, the consortium is also responsible for the Science Operations Centre and the Mission Operations Centre.

While the majority of the science observing time on CHEOPS is dedicated to a programme defined by the mission science team, $20 \%$ is available to the world-wide scientific community through ESA's Guest Observers Programme. The first of what are foreseen to be annual calls came out in March 2019, with proposal selection based on scientific merits by an independent committee. Around the time that routine observations start, a Discretionary Programme will open that will enable the observation of new targets to be made at any time.

The spacecraft will be lofted into a Sun-synchronous orbit of $700 \mathrm{~km}$ with a local time of the ascending node of 06:00 on a shared Soyuz launch from Europe's spaceport in Kourou, French Guiana.

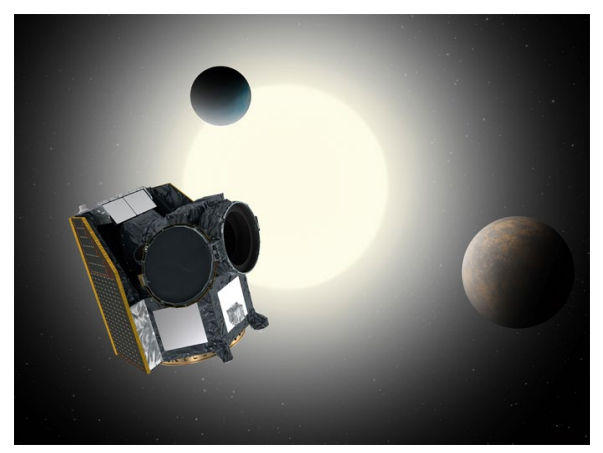

Fig. 1 | Artist's impression of CHEOPS. The CHEOPS spacecraft is shown against a background comprising a typical target exoplanetary system. Credit: ESA/ATG medialab

The nominal lifetime of the mission is 3.5 years, with a goal to extend it to 5 years. With the launch before the end of 2019 and a two-month, in-orbit commissioning period immediately following, the next year will be an exciting time for both the CHEOPS team and for exoplanet science more generally.

\section{K. G. Isaak ${ }^{1 *}$ and W. Benz ${ }^{2}$}

${ }^{1}$ Science Support Office, Science Directorate, ESTEC/European Space Agency, Noordwijk, the Netherlands. ${ }^{2}$ Physikalisches Institut,

Universität Bern, Bern, Switzerland.

*e-mail:kate.isaak@esa.int

Published online: 9 September 2019 https://doi.org/10.1038/s41550-019-0886-9

\section{References}

1. Mayor, M. \& Queloz, D. Nature 378, 355-359 (1995).

2. Benz, W., Ehrenreich, D. \& Isaak K. in Handbook of Exoplanets (eds Deeg, H. \& Belmonte, J.) 1257-1281 (Springer, 2018).

3. Beck, T. et al. Proc. SPIE 10562, 1056218 (2017).

4. Rando, N. et al. Proc. SPIE 10698, 106980K (2018).

\section{Acknowledgements}

CHEOPS is an ESA mission in ESA's Science Programme, implemented in partnership with Switzerland through the Swiss Space Office. The University of Bern leads a consortium of 11 ESA Member States (including Austria, Belgium, France, Germany, Hungary, Italy, Portugal, Spain, Sweden and the UK) who have made key contributions to the payload, to the Science Operations and Mission Operations centres, and to the CHEOPS Science Team. The authors gratefully acknowledge the contributions from members of the consortium, the Airbus Defence \& Space Spain (ASE) team priming the CHEOPS spacecraft activities, and ESA colleagues, and thank them for all of their efforts to make the CHEOPS mission a success. 